\title{
sciendo
}

Int. J. of Applied Mechanics and Engineering, 2020, vol.25, No.2, pp.142-156

DOI: $10.2478 /$ ijame-2020-0026

\section{OUTCOME OF NANOFLUID FLOW CONTAINING ARBITRARY SHAPE NANOPARTICLES INDUCED BY A PERMEABLE STRETCHING SHEET}

\author{
A. SARKAR* \\ Dept. of Mathematics, S.A. Jaipuria College \\ Kolkata 700005, West Bengal, INDIA \\ E-mail: amitformath@gmail.com \\ P.K. KUNDU \\ Dept. of Mathematics, Jadavpur University \\ Kolkata 700032, West Bengal, INDIA \\ E-mail: kunduprabir@yahoo.co.in
}

\begin{abstract}
In this work we have discussed the impact of thermal radiation on heat transfer to nanofluid flow over an unsteady permeable stretching sheet using various types of arbitrary shape nanoparticles of Copper $(\mathrm{Cu})$, Silver (Ag), Alumina $\left(\mathrm{Al}_{2} \mathrm{O}_{3}\right)$, and Titania Oxide $\left(\mathrm{TiO}_{2}\right)$ in the base fluid. Suitable transformations have been employed to build ODEs from the partial differential equations. Numerical results are therefore obtained particularly for cylindrical shape and spherical shape nanoparticles. Our analysis substantiates that the velocity and temperature profiles increases with enhanced thermal radiation parameter. Further, Nusselt number is more advanced for the nanofluid that contains cylindrical shape nanoparticles as compared to spherical shape nanoparticles.
\end{abstract}

Key words: nanofluid, heat transfer, boundary layer flow, thermal radiation, permeable stretching sheet.

2010 Mathematics Subject Classification. 76W05

\section{Introduction}

In the case of heat transfer, thermal conductivity of fluids deed as a clamorous part in various branches of engineering and science. Common fluids (e.g. water, toluene, oil etc.) which are usually used in power reproduction, cooling system, chemical production, micro-electronics etc., are relatively low thermal conductive and consequently need more advancement in the thermal conductive nature. Choi [1] designed the idea to upgrade the thermal conductivity of base liquids by suspending high thermal conductive diminutive metallic particles in it. Afterward, that idea was communicated by several researchers by taking various metallic, non metallic nanoparticles or their oxides or carbon nanotubes (CNT's). Sarkar et al. [2] studied the outcome of the bioconvection on a nanofluid that saturated in a non-Darcian porous medium. Raju and Sandeep [3] numerically survey the heat and momentum transport of $\mathrm{CoFe}_{2} \mathrm{O}_{4}$-water nanofluid which flows over a spinning cone. Chakraborty et al. [4] exploit Ag-water to improve the convective flow over an inclined plane in existence of non-Darcy porous medium and solar radiation. More significant works [5-8] on nanofluid can be found in open literature. The development of thermal conductivity of common fluid not only depend the appearance of nanoparticles but also their shapes and size [9]. Murshed et al. [10] examined that CNTs can attained better thermal conductivity (approximately six times) than metallic nanoparticles in nanofluids. Recently, flow description of MHD nanofluid carrying both cylindrical and spherical shape nanoparticles have been examined by Das et al. [11].

\footnotetext{
* To whom correspondence should be addressed
} 
Nanofluid flow over permeable stretching sheet offers wide utility, such as in nuclear reactors, space cooling, bio-medicine, heat pump and many others. Mandy [12] investigated mixed convection flow and heat transfer of nanofluids over an unsteady stretching sheet. Kundu and Sarkar [13] considered the out-turn of the multifarious slip on Casson nanofluid flow over an extending cylinder in presence of solar radiation. Hsiao [14] studied analytically the upshot of viscous dissipation on micropolar nanofluid towards a stretching sheet using finite difference method. Three dimensional MHD flow and mass transfer of Al-water in extending sheet was inspected thoroughly by Reddy and Chamkha [15]. More compatible studies can be found in recent works [16-18].

The impact of thermal radiation on the boundary layer flow has an appreciable contribution in the overall surface heat transfer in a situation where convective heat transfer coefficients are small. Radiation effects on convective flow have received a speedy on-going importance in the engineering field, especially electrical power generation, solar power technology, space technology and other industrial areas. In illumination of these various applications, Nayak et al. [19] originated the study of thermal radiation on nanofluid flow under MHD force convection in three dimensions. Later, Valipour et al. [20] diversify that study with CNT- water nanofluid. The effect of thermal radiation on $\mathrm{Al}_{2} \mathrm{O}_{3}$-EG nanofluid which flows through a porous and wavy channel was described by Zeeshan et al. [21]. Kumar et al. [22] assessed the mechanism of Joule heating on three dimensional Oldroyd B nanofluid by accounting the thermal radiation. More relevant surveys can be encountered in literature [23-26].

It can be almost definitely being said that the theme which has been diligently developed in this paper is not pondered over and has not been yet conveyed to anywhere. we have investigated the impact of thermal radiation on an unsteady boundary layer flow of $\mathrm{Al}_{2} \mathrm{O}_{3}$ - water nanofluid over a permeable stretching sheet in the presence of arbitrary shaped nanoparticles. The foremost partial differential equations are reduced into ordinary ones by similarity transformations, and then solved numerically. Numerical results of skin friction coefficient and heat transfer rate as well as the velocity and temperature distributions are discussed by means of graphs and tables.

\section{Mathematical analysis}

The most popular way in which convective heat transfer of nanofluids can be modeled is the single phase approach. The single phase approach involves the nanofluid as a homogeneous mixture of fluid and particles, whose thermal properties are taken as a function of both constituents and their concentrations. This type of model is used in the present study. We consider an unsteady laminar mixed convection flow of an incompressible electrically conducting nanofluid over a vertical permeable stretching sheet as portrayed in Fig.1. The stretching sheet is moving persistently in the positive direction of $x$-axis with a velocity $U_{w}(x, t)=\frac{a x}{1-c t}$ where $a, c$ are positive constants with dimension $\frac{1}{\text { time }}$. Also the surface of the sheet is heated to a variable temperature of the form $T_{w}(x, t)=T_{\infty}+\frac{b x}{(1-c t)^{2}}$ which is higher than the ambient temperature $T_{\infty}$ and $b$ is a constant with dimension temperature/length. The expressions for $U_{w}(x, t)$ and $T_{w}(x, t)$ are valid for time $t<c^{-1}$. The magnetic field $B=\frac{B_{0}}{\sqrt{1-c t}}$ is applied normal to the surface where $B_{0}$ is the initial intensity of the magnetic field. The induced magnetic field is assumed to be small. It is also assumed that there is no applied voltage. The base fluid is the water containing different kinds of nanoparticles: copper $(\mathrm{Cu})$, silver $(\mathrm{Ag})$, alumina $\left(\mathrm{Al}_{2} \mathrm{O}_{3}\right)$ and Titanium dioxide $\left(\mathrm{TiO}_{2}\right)$.

Under the above assumptions, the required leading equations can be written as [11, 13, 27] 


$$
\begin{aligned}
& \frac{\partial u}{\partial x}+\frac{\partial v}{\partial y}=0 \\
& \rho_{n f}\left(\frac{\partial u}{\partial t}+u \frac{\partial u}{\partial x}+v \frac{\partial u}{\partial y}\right)=\mu_{n f} \frac{\partial^{2} u}{\partial y^{2}}+g(\beta \rho)_{n f}\left(T-T_{\infty}\right)-\sigma B^{2} u \\
& \left(\rho C_{p}\right)_{n f}\left(\frac{\partial T}{\partial t}+u \frac{\partial T}{\partial x}+v \frac{\partial T}{\partial y}\right)=\kappa_{n f} \frac{\partial^{2} T}{\partial y^{2}}-\frac{\partial q_{r}}{\partial y}
\end{aligned}
$$

where $(x, y)$ denotes the Cartesian coordinates along the sheet and normal to it, $u$ and $v$ are the velocity components of the nanofluid in the $x$ and $y$ directions respectively, $g$ is acceleration due to gravity, $T$ is the temperature of the fluid within the boundary layer, $q_{r}$ is the radiative heat flux in the vertical direction of $x$ - axis, $\rho_{n f}$ is the effective density of the nanofluid, $\left(\rho C_{p}\right)_{n f}$ is the heat capacitance, $\beta_{n f}$ is the thermal expansion coefficient of the nanofluid. The quantities ( $)_{n f}$ are defined as

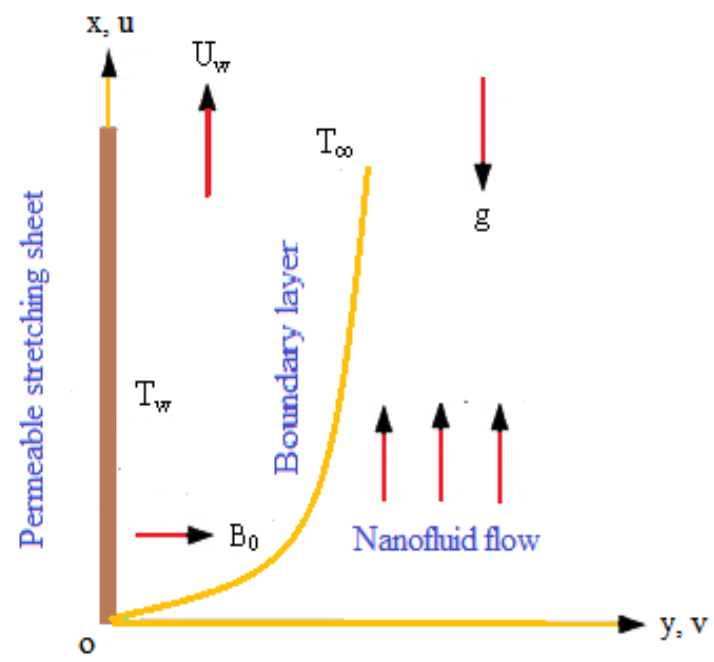

Fig.1. Physical model and coordinate system.

$$
\begin{aligned}
& \rho_{n f}=(1-\phi) \rho_{f}+\phi \rho_{s}, \quad\left(\rho C_{p}\right)_{n f}=(1-\phi)\left(\rho C_{p}\right)_{f}+\phi\left(\rho C_{p}\right)_{s}, \\
& (\rho \beta)_{n f}=(1-\phi)(\rho \beta)_{f}+\phi(\rho \beta)_{s}
\end{aligned}
$$

where $\phi$ is the solid volume fraction, ()$_{f}$ and ()$_{S}$ denote the basic fluid and solid fractions, respectively. Furthermore, the effective dynamic viscosity of nanofluids $\mu_{n f}$ is given by [28]

$$
\mu_{n f}=\mu_{f}(1-\phi)^{-2.5}
$$


On the other hand, the thermal conductivity of nanofluid $\kappa_{n f}$ is influenced by different shape of particles. Numerous studies have found that rod-shaped nanoparticles, such as CNT, remove more heat than spherical shaped nanoparticles [29, 30]. This may be due to the fact that rod-shaped particles have a larger aspect ratio (the ratio between a particle's surface area to volume) than spherical shape nanoparticles. The effective thermal conductivity $\kappa_{n f}$ of the nanofluid for arbitrary shape nanoparticles according to Hamilton and Crosser [31] model is given by

$$
\kappa_{n f}=\kappa_{f}\left[\frac{\kappa_{s}+(\mathrm{n}-1) \kappa_{f}-(\mathrm{n}-1) \phi\left(\kappa_{f}-\kappa_{s}\right)}{\kappa_{s}+(\mathrm{n}-1) \kappa_{f}+\phi\left(\kappa_{f}-\kappa_{s}\right)}\right]
$$

where $n$ is the empirical shape factor for nanoparticles and is defined as $n=3 / \psi$ and $\psi$ is the ratio of the sphericity defined as the ratio of the surface area of a sphere (with the same volume as the given particle) to the surface area of the particle. In particular $n=3$ for spherical shaped nanoparticles and $n=4.5$ for cylindrical (nanotube) shaped nanoparticles.

On using the Rosseland approximation for radiation [32], the radiative heat flux is simplified as

$$
q_{r}=-\frac{4 \sigma^{*}}{3 k^{*}} \frac{\partial T^{4}}{\partial y}
$$

where $\sigma^{*}$ is the Stefan Boltzmann constant and $k^{*}$ is the Rosseland mean absorption coefficient. Substituting Eq.(2.6) in the central Eq.(2.3), we get

$$
\left(\rho C_{p}\right)_{n f}\left(\frac{\partial T}{\partial t}+u \frac{\partial T}{\partial x}+v \frac{\partial T}{\partial y}\right)=\kappa_{n f} \frac{\partial^{2} T}{\partial y^{2}}+\frac{16 \sigma^{*}}{3 k^{*}} \frac{\partial}{\partial y}\left(T^{3} \frac{\partial T}{\partial y}\right) .
$$

The appropriate boundary conditions of the Eqs (2.2) and (2.7) are as follows

$$
\left.\begin{array}{l}
u=U_{w}, \quad v=v_{w}, \quad T=T_{w} \quad \text { at } \quad y=0, \\
u \rightarrow 0, \quad T \rightarrow T_{\infty} \quad \text { as } \quad y \rightarrow \infty
\end{array}\right\}
$$

where $v_{w}$ is the suction/injection velocity.

As mentioned by $[11,13,27]$, we introduce the following similarity variables

$$
\eta=\sqrt{\frac{a}{v_{f}(1-c t)}} y, \quad \psi=\sqrt{\frac{a v_{f}}{(1-c t)}} x f(\eta), \quad \theta(\eta)=\frac{T-T_{\infty}}{T_{w}-T_{\infty}}
$$

where $f(\eta)$ is the dimensionless stream function, $\theta(\eta)$ is the dimensionless temperature. Further, $\psi(x, y)$ is the stream function which is defined in the usual way as $u=\frac{\partial \psi}{\partial y}, v=-\frac{\partial \psi}{\partial x}$ and so Eq.(2.1) satisfies identically. Thus the velocity components $u$ and $v$ in terms of the stream function $\psi$ are given by

$$
u=\frac{a x}{1-c t} f^{\prime}(\eta), \quad v=-\sqrt{\frac{a v_{f}}{1-c t}} f(\eta)
$$

where the prime denotes differentiation with respect to $\eta$. 
Now on substituting Eq.(2.10) into Eqs (2.2) and (2.7), we get the following non-linear ordinary differential equations

$$
\begin{aligned}
& \frac{\mu_{n f}}{\mu_{f}} f^{\prime \prime \prime}+\left(1-\phi+\phi \frac{\rho_{s}}{\rho_{f}}\right)\left\{f^{\prime 2}-f f^{\prime \prime}-S\left(f^{\prime}+\frac{1}{2} \eta f^{\prime \prime}\right)\right\}-M^{2} f^{\prime}+\left\{1-\phi+\phi \frac{(\rho \beta)_{s}}{(\rho \beta)_{f}}\right\} \gamma \theta=0 \\
& \frac{\kappa_{n f}}{\kappa_{f}} \theta^{\prime \prime}-\operatorname{Pr}\left\{1-\phi+\phi \frac{\left(\rho C_{p}\right)_{s}}{\left(\rho C_{p}\right)_{f}}\right\}\left[f^{\prime} \theta-f \theta^{\prime}+S\left(2 \theta+\frac{1}{2} \eta \theta^{\prime}\right)\right]+(4 N / 3)\left[\left\{1+\left(T_{r}-1\right) \theta\right\}^{3} \theta^{\prime}\right]^{\prime}=0 .
\end{aligned}
$$

The boundary conditions (2.8) then turn into

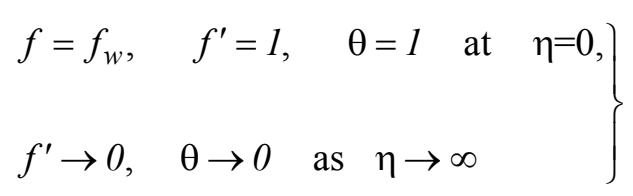

where $M=B_{0} \sqrt{\frac{\sigma}{a \rho_{f}}}$ is the magnetic field parameter, $S=\frac{c}{a}$ is the unsteadiness parameter, $\gamma=\frac{g \beta_{f}\left(T_{w}-T_{\infty}\right)}{a U_{w}}$ is the mixed convection parameter, $N=\frac{4 \sigma^{*} T_{\infty}{ }^{3}}{k_{m} k^{*}}$ is the conduction radiation parameter, $T_{r}=\frac{T_{w}}{T_{\infty}}$ is the temperature ratio parameter, $f_{w}=-v_{w} / \sqrt{\frac{a v_{f}}{1-c t}}$ is the suction/injection parameter $\left(f_{w}>0\right.$ represents suction, $f_{w}<0$ represents injection and $f_{w}=0$ corresponds to an impermeable surface) and $\operatorname{Pr}=\frac{v_{f}\left(\rho \mathrm{C}_{p}\right)_{f}}{\kappa_{f}}$ is the Prandtl number.

It should be noted that, in this study, the quantities of practical interest are the skin friction coefficient $C_{f}$ and the Nusselt number $\mathrm{Nu}$ which are defined in non-dimensional form as

$$
C_{f}=-\frac{\mu_{n f}}{\rho_{f} U_{w}^{2}}\left(\frac{\partial u}{\partial y}\right)_{y=0}=-\frac{\mu_{n f}}{\mu_{f}} \operatorname{Re}_{x}^{-1 / 2} f^{\prime \prime}(0),
$$

or, $\quad C_{f} r=C_{f} \operatorname{Re}_{x}^{1 / 2}=-\frac{\mu_{n f}}{\mu_{f}} f^{\prime \prime}(0)$

and $\left.\quad \mathrm{Nu}=-\frac{x \kappa_{n f}}{\kappa_{f}\left(T_{w}-T_{\infty}\right)}\left(\frac{\partial T}{\partial y}\right)_{y=0}=-\frac{\kappa_{n f}}{\kappa_{f}}\left[1+\frac{4 N}{3}\left\{1+\left(T_{r}-1\right)\right) \theta(0)\right\}^{3}\right] \operatorname{Re}_{x}^{1 / 2} \theta^{\prime}(0)$,

or, $\left.\quad \mathrm{Nur}=\mathrm{NuRe}_{x}^{-1 / 2}=-\frac{\kappa_{n f}}{\kappa_{f}}\left[1+\frac{4 N}{3}\left\{1+\left(T_{r}-1\right)\right) \theta(0)\right\}^{3}\right] \theta^{\prime}(0)$

where $C_{f} r$ is the reduced local skin friction coefficient, Nur is the reduced local Nusselt number and $\operatorname{Re}_{x}=\frac{U_{w} x}{v_{f}}$ is the local Reynolds number based on the stretching velocity $U_{w}$. 


\section{Method of solution}

To solve the coupled non-linear two-point boundary value problem, Eqs (2.11) and (2.12) with respect to the boundary conditions (2.13) are solved numerically by employing shooting technique with RK4 integration scheme. The computer program of the numerical method is executed in MATHEMATICA 8 running on a PC. A step size of $\Delta \eta=0.001$ is selected to be satisfactory for a convergence criterion of $10^{-8}$ in nearly all cases. The value of $\eta_{\infty}$ is found to each iteration loop by assignment statement $\eta_{\infty}=\eta_{\infty}+\Delta \eta$. The maximum value of $\eta_{\infty}$ is determined to each group of pertinent parameters when no considerable variation in velocity, temperature etc. occurs. In order to validate our method, we have compared the results of $-\theta^{\prime}(0)$ for various values of $\operatorname{Pr}$ and $\gamma$ (Tab.1) for pure water in absence of magnetic field and thermal radiation with those of Das et al. [11] and Mandy [12] and found them in excellent agreement.

Table 1. Comparison of the current results with previous published work.

\begin{tabular}{|c|c|c|c|c|}
\hline \multirow{2}{*}{$\gamma$} & \multirow{2}{*}{$\operatorname{Pr}$} & \multicolumn{3}{|c|}{$-\theta^{\prime}(0)$} \\
\cline { 3 - 5 } & & Mandy [12] & Das et al. $[11]$ & Present result \\
\hline 0 & 0.01 & 0.01999 & 0.019706 & 0.01970610 \\
\hline 1.0 & 1.0 & 1.00000 & 1.000000 & 1.00000000 \\
\hline 2.0 & 3.0 & 1.92368 & 1.923682 & 1.92368186 \\
\hline 3.0 & 7.0 & 3.07224 & 3.072250 & 3.07225013 \\
\hline
\end{tabular}

\section{Results and discussion}

In this section, we have discoursed the influences of various types of nanoparticles on the distribution of $f^{\prime}$ and $\theta$ as well as $C_{f} r$ and Nur, for several values of $N, f_{w}$ and volume fraction of nanoparticles $\phi$ at $M=0.4, S=0.5$ and $\operatorname{Pr}=6.785$. Four different types of nanoparticles, viz Copper $(\mathrm{Cu})$, Silver (Ag), Alumina $\left(\mathrm{Al}_{2} \mathrm{O}_{3}\right)$ and Titanium Oxide $\left(\mathrm{TiO}_{2}\right)$ are considered by using water based fluid, where thermophysical properties of fluid and these nanoparticles are given in Tab.2.

Table 2. Thermophysical properties of base fluid and nanoparticles.

\begin{tabular}{|c|c|c|c|c|c|}
\hline $\begin{array}{c}\text { Physical } \\
\text { properties }\end{array}$ & Water & Copper & Silver & Alumina & Titanate \\
\hline$C_{p}(J / \mathrm{kg} \mathrm{K})$ & 4179 & 385 & 235 & 765 & 686.2 \\
\hline$\rho\left(\mathrm{kg} / \mathrm{m}^{3}\right)$ & 997.1 & 8933 & 10500 & 3970 & 4250 \\
\hline$\kappa(W / \mathrm{m} \mathrm{K})$ & 0.613 & 401 & 429 & 40 & 8.9538 \\
\hline$\beta \times 10^{-5}\left(K^{-1}\right)$ & 21.0 & 1.67 & 1.89 & 0.85 & 0.90 \\
\hline
\end{tabular}

\subsection{Consequence of radiation parameter $N$}

The variations of $f^{\prime}$ against transverse coordinate $\eta$ are shown in Fig. 2 for different values of $N$ for $\mathrm{Al}_{2} \mathrm{O}_{3}$ - water nanofluid containing both type (spherical and cylindrical) of nanoparticles. The outcome indicates that the raising value of the parameter $N$, increases $f^{\prime}$ within the boundary region of the permeable plate. Thus, the momentum boundary layer thickness increases. It is worthy to mention that these profiles 
asymptotically satisfy the boundary conditions at infinity, which support the numerical results obtained. Table 3 shows up that for $\mathrm{Al}_{2} \mathrm{O}_{3}$ - water nanofluid flow $C_{f} r$ reduces by $3.71 \%$ and $3.58 \%$ in existence of spherical and cylindrical shape nanoparticles respectively when $N=3$. Clearly the reducing rate is higher for spherical shape nanoparticles as compared to cylindrical shape. On the other hand, the values of $C_{f} r$ is

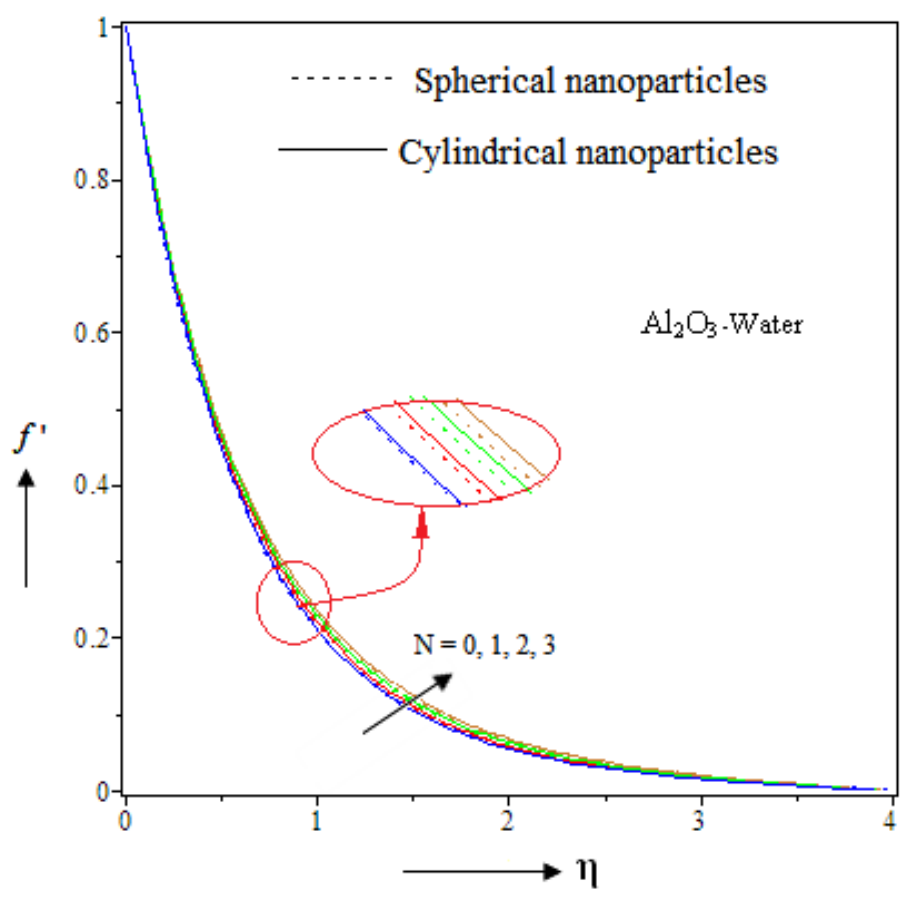

Fig.2. Velocity profiles for various values of $N$.

higher for cylindrical shape nanoparticles by $0.44 \%$ than that of spherical shape nanoparticles. Pictorial description of $\theta$ is presented in Fig. 3 for $\mathrm{Al}_{2} \mathrm{O}_{3}$ - water nanofluid. It is observed that $\theta$ increases as $N$ because of thermal radiation boosts conduction effect of the nanofluid. Again $\theta$ possesses high value for nanofluid flow containing cylindrical shape nanoparticles. One may observed from Table 3 that Nur increases with increase in the values of $N$ in presence of both types of nanoparticles but better heat transfer at the surface occurs for nanofluid containing cylindrical nanoparticles. For $\mathrm{Al}_{2} \mathrm{O}_{3}$ - water nanofluid, Nur increases by $111.86 \%$ for spherical nanoparticles and $110.44 \%$ for cylindrical nanoparticles.

Table 3. Values of $C_{f} r$ for various values of $N$.

\begin{tabular}{|c|c|c|c|c|}
\hline \multirow{2}{*}{$N$} & \multicolumn{2}{|c|}{$C_{f} r$} & \multicolumn{2}{c|}{ Nur } \\
\cline { 2 - 5 } & $\begin{array}{c}\text { Spherical } \\
\text { nanoparticles }\end{array}$ & $\begin{array}{c}\text { Cylindrical } \\
\text { nanoparticles }\end{array}$ & $\begin{array}{c}\text { Spherical } \\
\text { nanoparticles }\end{array}$ & $\begin{array}{c}\text { Cylindrical } \\
\text { nanoparticles }\end{array}$ \\
\hline 0.0 & 2.4628408 & 2.47063099 & 0.7161993 & 0.80135600 \\
\hline 1.0 & 2.41629029 & 2.42614135 & 1.06980912 & 1.19235408 \\
\hline 2.0 & 2.38980214 & 2.40028512 & 1.31648026 & 1.46494313 \\
\hline 3.0 & 2.37142990 & 2.38212152 & 1.51724024 & 1.68638941 \\
\hline
\end{tabular}

\subsection{Consequence of nanoparticle volume fraction $\phi$}

The impact of $\phi$ on $f^{\prime}$ for $\mathrm{Al}_{2} \mathrm{O}_{3}$ - water nanofluid is found opposite of that of $N$ (Fig.4). It is further observed that the influence of $\phi$ is independent on the nature of the shape of nanoparticles. One may 
perceive from Tab.4 that $C_{f} r$ of $\mathrm{Al}_{2} \mathrm{O}_{3}$ - water nanofluid enhances $100.89 \%$ in presence of spherical shape nanoparticles and $101.74 \%$ for cylindrical shape

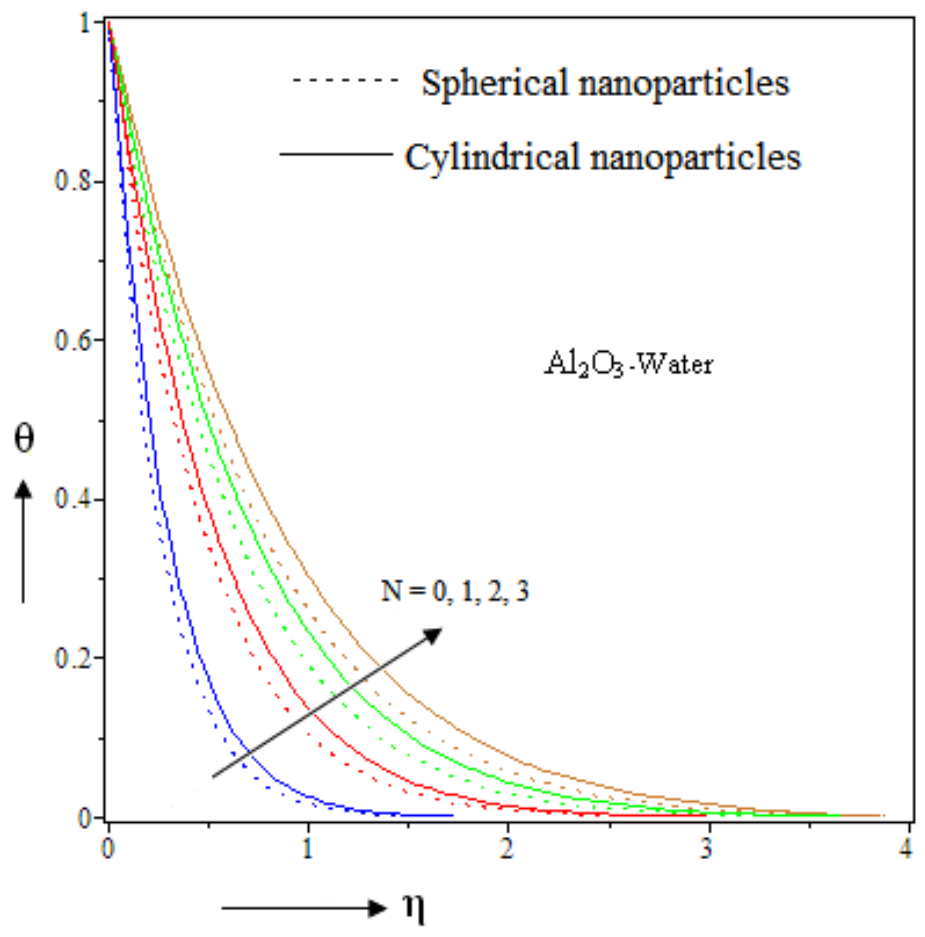

Fig.3. Temperature profiles for various values of $N$.

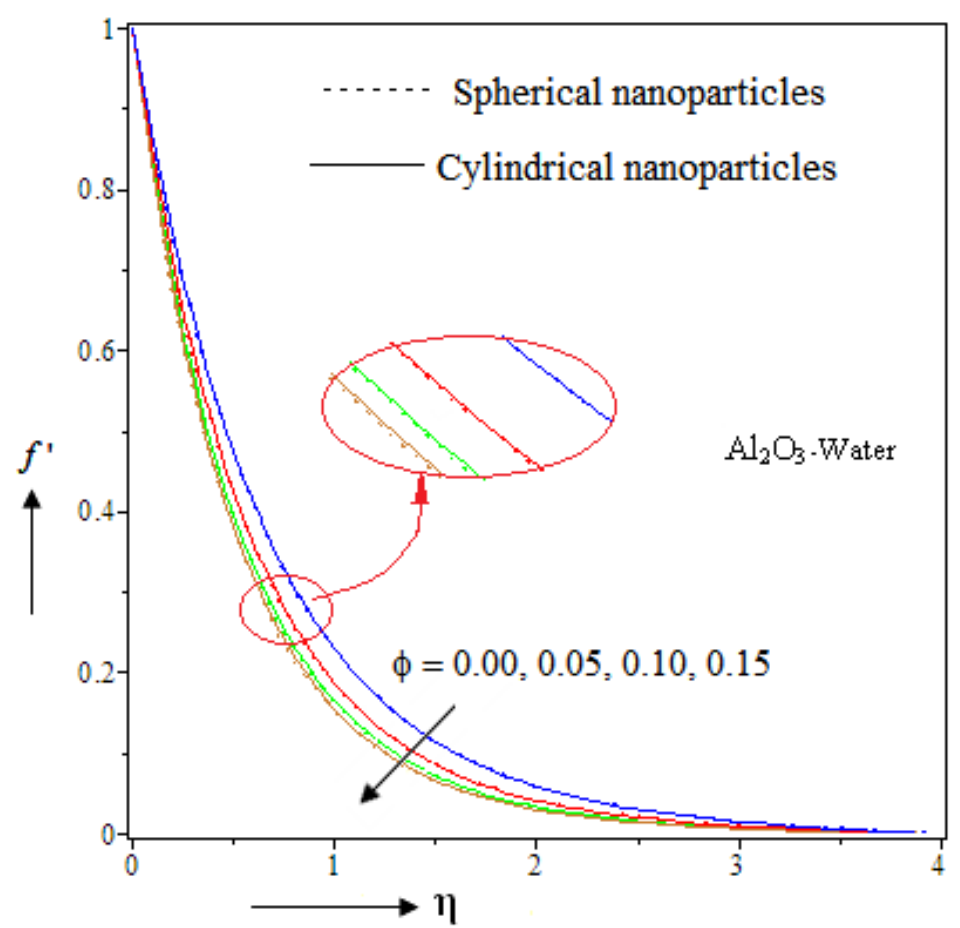

Fig.4. Velocity profiles for various values of $\phi$. 
nanoparticles as $\phi$ increases up to 0.15 . It is worth mentioning that the values of $C_{f} r$ is utmost for cylindrical shape nanoparticles by $0.34 \%$ than that of spherical shape nanoparticles. The presence of nanoparticles leads to an increase in $\theta$ in the boundary region of heated plate (Fig.5) and subsequently boosts the thickness of the thermal boundary layer. This result is correlated to the enrichment of thermal conductivity of the nanofluid. Truly, high thermal conductivity is escorted by large values of thermal diffusivity which

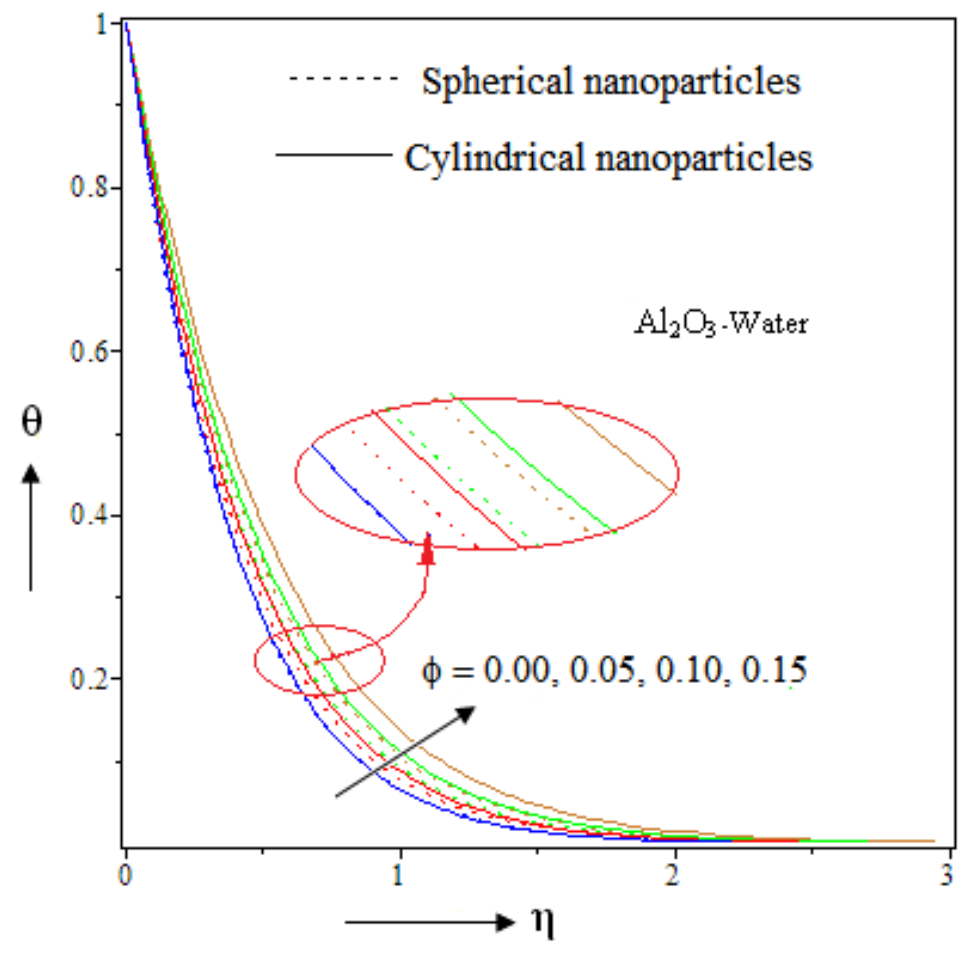

Fig.5. Temperature profiles for various values of $\phi$.

causes a drop in the temperature gradients and accordingly thermal boundary layer thickness increases as established. This enhancement in boundary layer thickness reduces Nur (Tab.4). So, one can interpret that Nur decreases with increasing $\phi$. Additionally, the influence of $\phi$ is more effective for cylindrical shape nanoparticles. The numerical upshot received from Tab.4 substantiate that Nur of $\mathrm{Al}_{2} \mathrm{O}_{3}$ - water nanofluid drops off by $28.87 \%$ for spherical shape nanoparticles and $19.39 \%$ for cylindrical shape nanoparticles when $\phi=0.15$.

Table 4. Values of $C_{f} r$ for various values of $\phi$.

\begin{tabular}{|c|c|c|c|c|}
\hline \multirow{2}{*}{$\phi$} & \multicolumn{2}{|c|}{$C_{f} r$} & \multicolumn{2}{c|}{ Nur } \\
\cline { 2 - 5 } & $\begin{array}{c}\text { Spherical } \\
\text { nanoparticles }\end{array}$ & $\begin{array}{c}\text { Cylindrical } \\
\text { nanoparticles }\end{array}$ & $\begin{array}{c}\text { Spherical } \\
\text { nanoparticles }\end{array}$ & $\begin{array}{c}\text { Cylindrical } \\
\text { nanoparticles }\end{array}$ \\
\hline 0.00 & 1.48557573 & 1.48557573 & 1.76775847 & 1.76775847 \\
\hline 0.05 & 1.97121120 & 1.97549694 & 1.56826641 & 1.64388946 \\
\hline 0.10 & 2.46674428 & 2.47524047 & 1.40057165 & 1.53019869 \\
\hline 0.15 & 2.98438560 & 2.99696566 & 1.25746228 & 1.42493589 \\
\hline
\end{tabular}




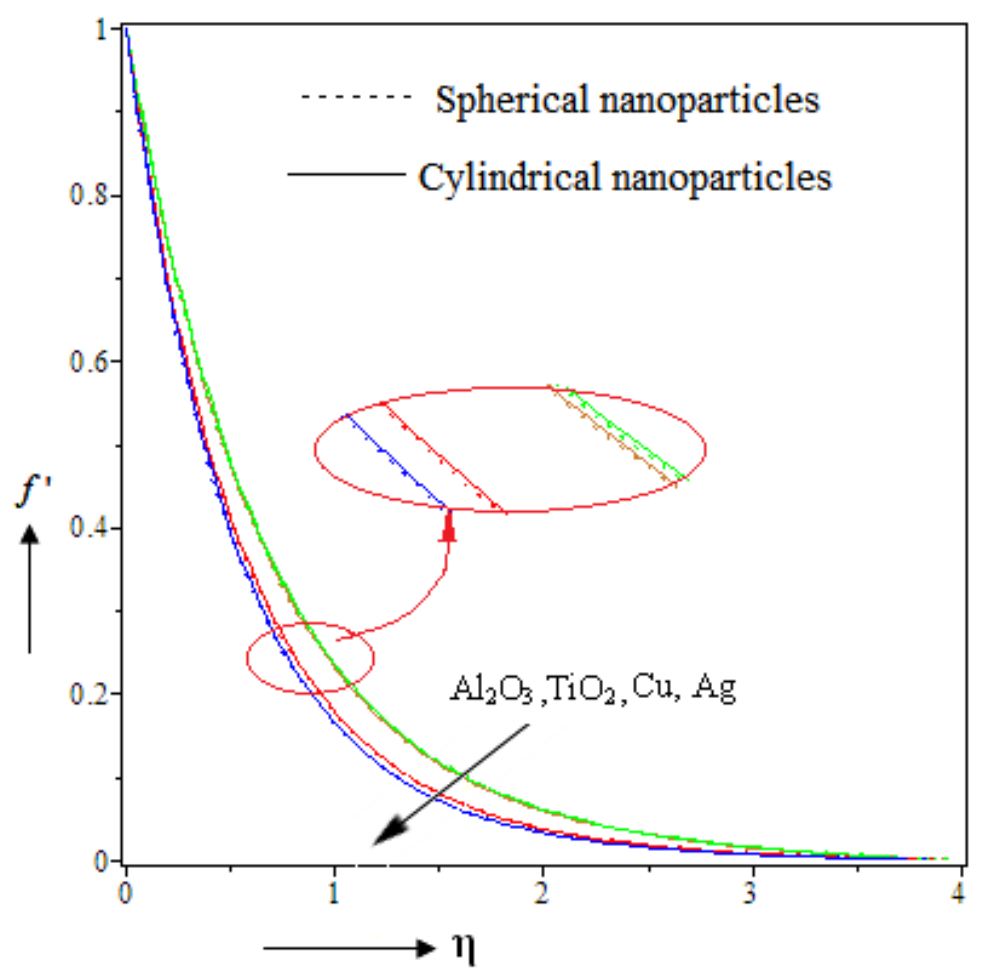

Fig.6. Velocity profiles for different types of nanoparticles.

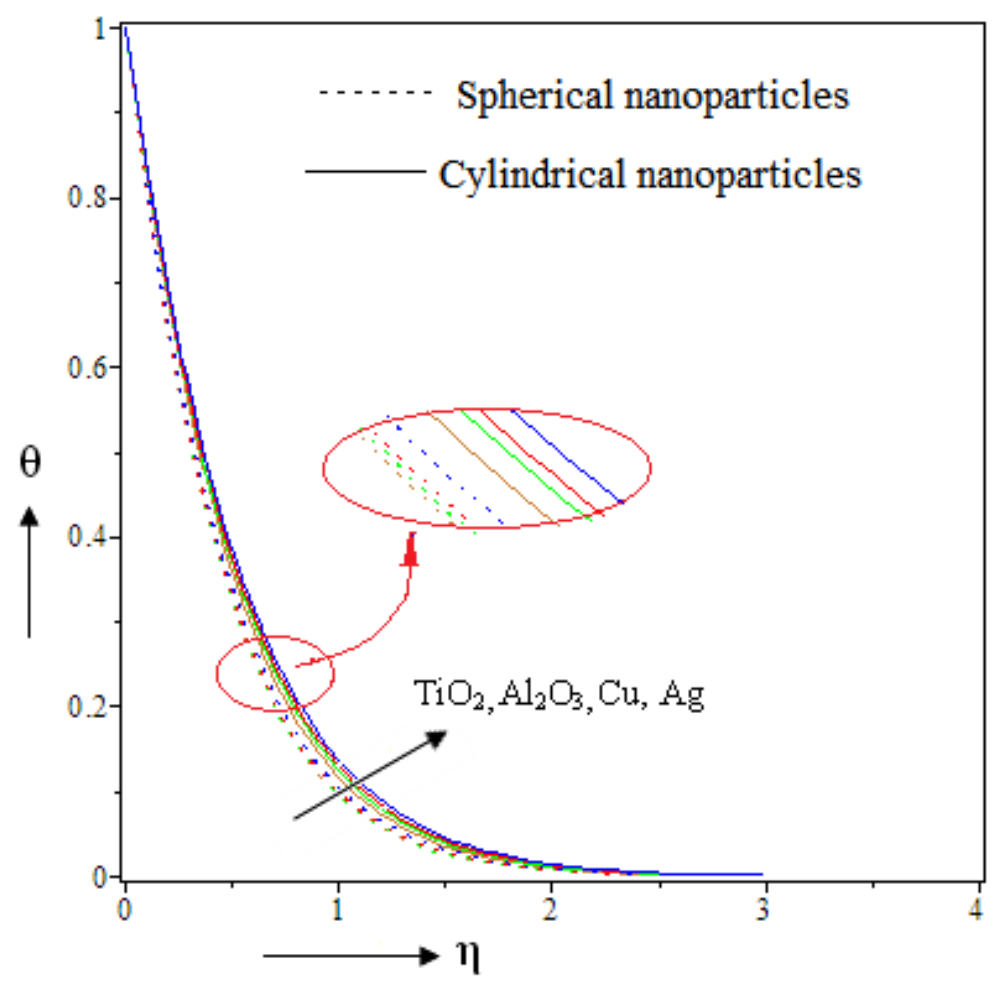

Fig.7. Temperature profiles for different nanoparticles.

It is further noticed that the influence of $\phi$ is more effective for cylindrical nanoparticles and is elevated by $9.26 \%$ (approximately) as compared to spherical shape nanoparticles. 


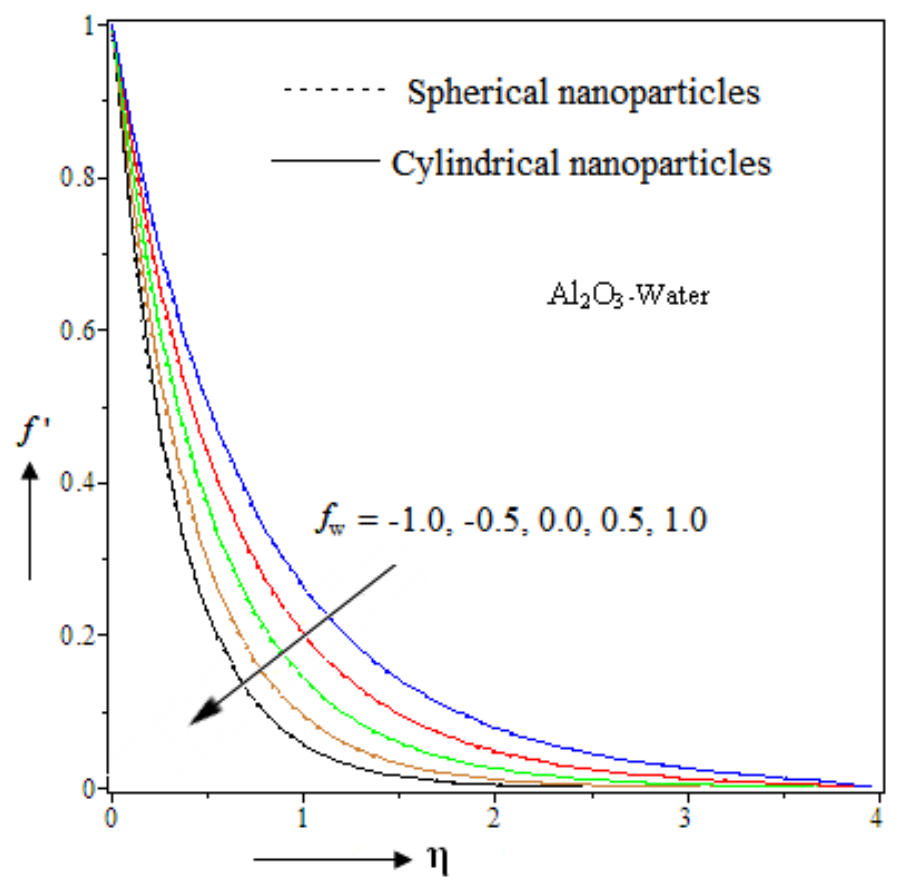

Fig.8. Velocity profiles for various values of $f_{w}$.

\subsection{Consequence of different nanoparticles}

It is monitored from Fig.6 that the nanofluid flow drops off in presence of various nanoparticles and upshot is more for high thermal conductive nanoparticles like $\mathrm{Cu}$ and $\mathrm{Ag}$. Additionally, throughout the boundary layer, $f^{\prime}$ decreases monotonically with the increase of the boundary layer coordinate $\eta$. The effect of various types of nanoparticles on $\theta$ is watched out in Fig.7. The outcome of the figure demonstrates that $\theta$ is significantly affected by the addition of different type of nanoparticles in water and is prominent for cylindrical shape nanoparticles.

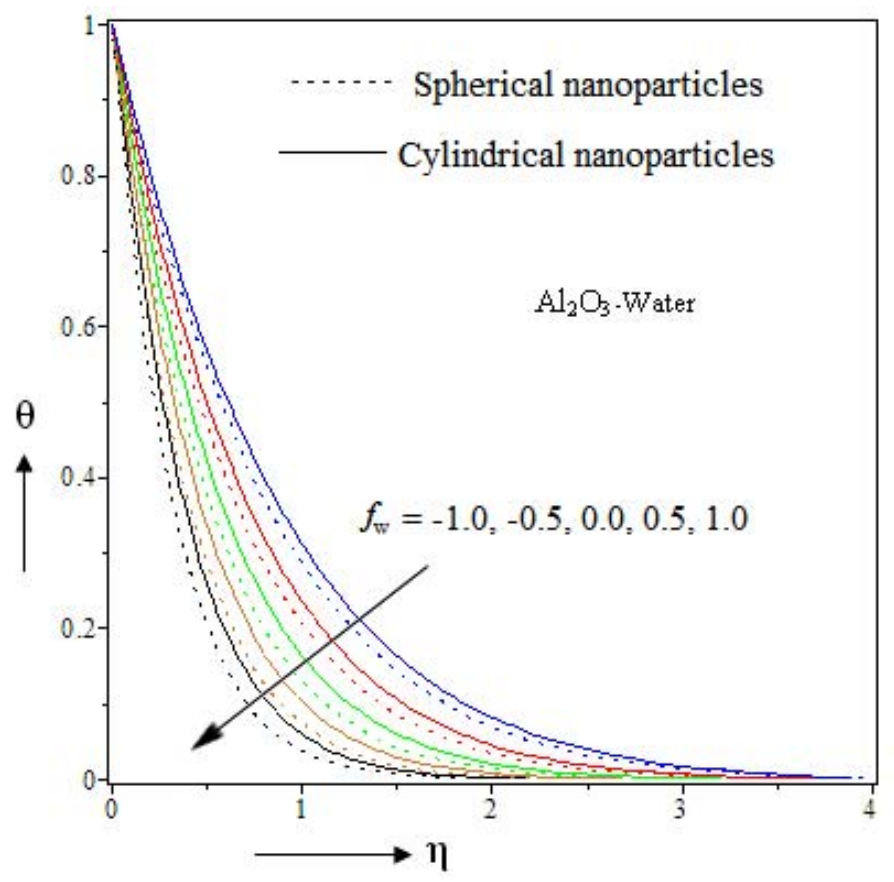

Fig.9. Temperature profiles for various values of $f_{w}$. 
Moreover, one can see that the temperature profiles of $\mathrm{Ag}$ - water and $\mathrm{Cu}$ - water are higher than the other nanofluids. This is due to the existence of high thermal conductivity of nanoparticles Ag and $\mathrm{Cu}$. Also, the heat transport performance of the nanofluid enhances in presence of cylindrical nanoparticles as highlighted in Tab.5. An important observation from the Tab.5 is that Nur achieves its maximum value for nanofluid containing $\mathrm{Al}_{2} \mathrm{O}_{3}$ nanoparticles.

Table 5. Values of $C_{f} r$ for various types of nanoparticles.

\begin{tabular}{|c|c|c|c|c|}
\hline \multirow{2}{*}{$\begin{array}{c}\text { Types of } \\
\text { nanoparticles }\end{array}$} & \multicolumn{2}{|c|}{$C_{f} r$} & \multicolumn{2}{c|}{ Nur } \\
\cline { 2 - 5 } & $\begin{array}{c}\text { Spherical } \\
\text { nanoparticles }\end{array}$ & $\begin{array}{c}\text { Cylindrical } \\
\text { nanoparticles }\end{array}$ & $\begin{array}{c}\text { Spherical } \\
\text { nanoparticles }\end{array}$ & $\begin{array}{c}\text { Cylindrical } \\
\text { nanoparticles }\end{array}$ \\
\hline $\mathrm{Ag}$ & 2.47524177 & 2.46679633 & 0.28622367 & 0.29906392 \\
\hline $\mathrm{Cu}$ & 2.35109775 & 2.34290607 & 0.28483255 & 0.29758821 \\
\hline $\mathrm{Al}_{2} \mathrm{O}_{3}$ & 1.93214421 & 1.92475283 & 0.28845127 & 0.30082998 \\
\hline $\mathrm{TiO}_{2}$ & 1.95728533 & 1.95150626 & 0.28739669 & 0.29716714 \\
\hline
\end{tabular}

\subsection{Consequence of suction/injection parameter $f_{w}$}

Figure 8 enlighten us about the impact of $f_{w}$ on $\mathrm{Al}_{2} \mathrm{O}_{3}$ - water nanofluid velocity considering both types of nanoparticles. It illustrates that $f^{\prime}$ decreases with the increase of suction parameter $f_{w}(>0)$ whereas opposite effect is detected for injection parameter $f_{w}(<0)$. It should be noted that the effect of $f_{w}$ is independent on nature of the shape of nanoparticles. Table 6 highlights that $C_{f} r$ enhances by $47.14 \%$ with the influence of suction parameter $f_{w}(>0)$ but reduce by $31.50 \%$ for injection parameter $f_{w}(<0)$ for $\mathrm{Al}_{2} \mathrm{O}_{3}$-water nanofluid containing spherical shape nanoparticles. The same is achieved by $47.23 \%$ and $31.58 \%$ respectively for nanofluid containing cylindrical nanoparticles. It is seen from the Fig.9 that the temperature distribution near the permeable boundary surface decreases with increasing values of suction parameter $f_{w}(>0)$. But the effect is contrary for injection parameter $f_{w}(<0)$. Table 6 informs us that Nur increases by $47.16 \%$ for spherical shape nanoparticles as suction parameter $f_{w}(>0)$ increases up to $1.0 \%$ where as the effect is reverse for injection parameter and it diminishes by $31.54 \%$ and $34.77 \%$ respectively.

Table 6. Values of $C_{f} r$ for various values of $f_{w}$.

\begin{tabular}{|c|c|c|c|c|}
\hline \multirow{2}{*}{$f_{w}$} & \multicolumn{2}{|c|}{$C_{f} r$} & \multicolumn{2}{c|}{ Nur } \\
\cline { 2 - 5 } & $\begin{array}{c}\text { Spherical } \\
\text { nanoparticles }\end{array}$ & $\begin{array}{c}\text { Cylindrical } \\
\text { nanoparticles }\end{array}$ & $\begin{array}{c}\text { Spherical } \\
\text { nanoparticles }\end{array}$ & $\begin{array}{c}\text { Cylindrical } \\
\text { nanoparticles }\end{array}$ \\
\hline-1.0 & 2.46754866 & 2.4701166 & 0.60097377 & 0.65005954 \\
\hline-0.5 & 2.96871505 & 2.97352077 & 0.72318959 & 0.8009394 \\
\hline 0.0 & 3.60242224 & 3.61047545 & 0.87789373 & 0.99835239 \\
\hline 0.5 & 4.38098063 & 4.39275475 & 1.06785033 & 1.24631925 \\
\hline 1.0 & 5.3007724 & 5.31572936 & 1.29190882 & 1.54099846 \\
\hline
\end{tabular}

\section{Final remarks}

Based on whole investigation some important findings are listed as follows:

* $f^{\prime}$ enhances by $N$ and injection parameter. But opposite outcome is noticed for $\phi$ and suction parameter. 
* Again $\theta$ amplifies in response to $N, \phi$ and injection parameter whereas opposite trend is observed for suction parameter.

* A novel characteristic that comes into view is that, the rate of heat transfer of $\mathrm{Al}_{2} \mathrm{O}_{3}$ - water nanofluid is high for cylindrical shape nanoparticles as compared to nanofluid containing spherical shape nanoparticles

* $\quad C_{f} r$ as well as Nur increases due to suction but converse effect occurs for injection.

\section{Nomenclature}

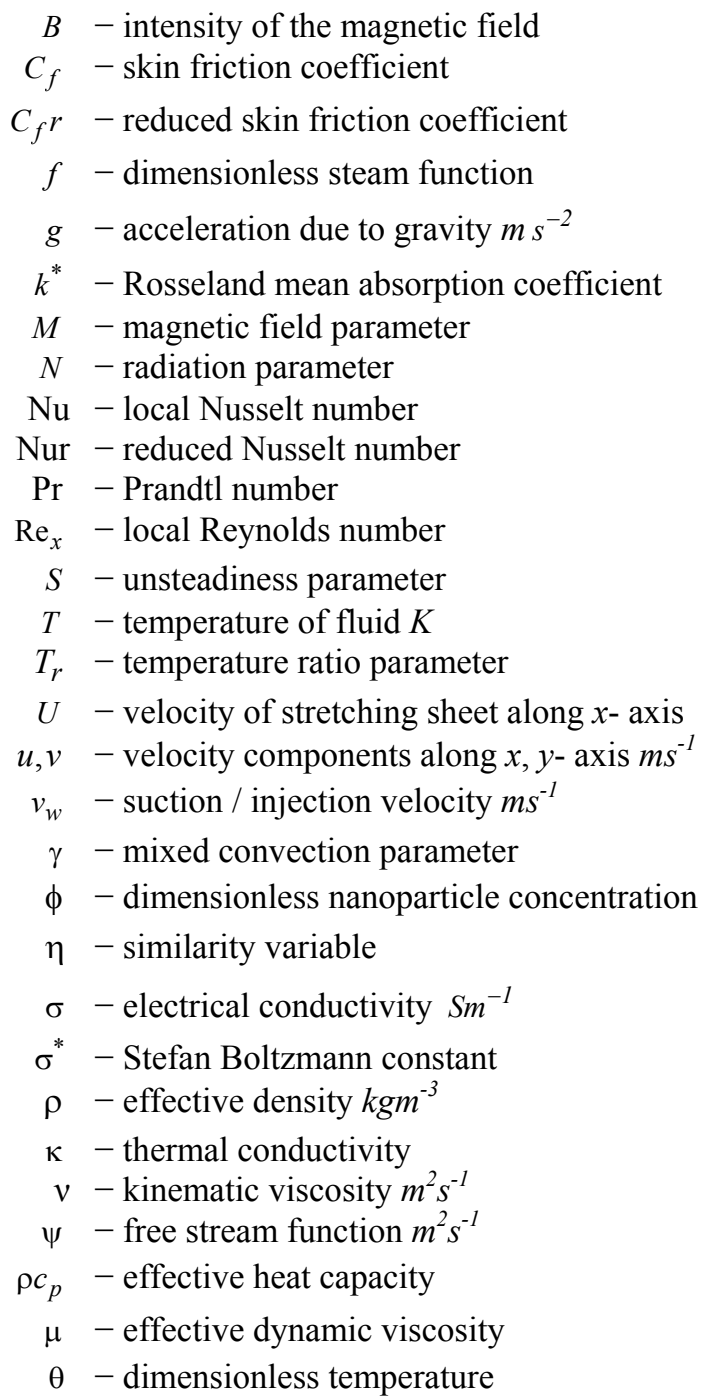

\section{Subscript}

$f$ - base fluid

$f_{w}$ - suction / injection parameter

$n f$ - nanofluid

$q_{r}$ - radiative heat flux

$s$ - nanoparticles

$w$ - condition on the sheet 
$\beta-$ volume expansion coefficient

$\theta$ - dimensionless temperature

$\infty-$ condition far away from the sheet

' - differentiation with respect to $\eta$

\section{References}

[1] Choi S.U.S. (1995): Enhancing thermal conductivity of fluids with nanoparticles. - Dev. Appl. Non-Newton Flows, vol.66, 99.

[2] Sarkar A., Das K. and Kundu P.K. (2016): On the onset of bioconvection in nanofluid containing gyrotactic microorganisms and nanoparticles saturating a non-Darcian porous medium. - J. Mol. Liq., vol.223, pp.725-733.

[3] Raju C.S.K. and Sandeep N. (2017): Unsteady Casson nanofluid flow over a rotating cone in a rotating frame filled with ferrous nanoparticles: A numerical study. - J. Magnetism and Magnetic Materials, vol.421, pp.216-224.

[4] Chakraborty T., Das K. and Kundu P.K. (2017): Ag-water nanofluid flow over an inclined porous plate embedded in a non-Darcy porous medium due to solar radiation. - J. Mech. Sci. Tech., vol.31, No.5, pp.2443-2449.

[5] Sheikholeslami M. (2018): Numerical investigation for $\mathrm{CuO}-\mathrm{H}_{2} \mathrm{O}$ nanofluid flow in a porous channel with magnetic field using mesoscopic method. - J. Mol. Liq., vol.249, pp.739-746.

[6] Sheikholeslami M. and Shehzad S.A. (2018): Numerical analysis of $\mathrm{Fe}_{3} \mathrm{O}_{4}-\mathrm{H}_{2} \mathrm{O}$ nanofluid flow in permeable media under the effect of external magnetic source. - Int. J. Heat Mass Trans., vol.118, pp.182-192.

[7] Salari M., Malekshah E.H. and Malekshah M.H. (2018): Natural convection in a rectangular enclosure filled by two immiscible fluids of air and $\mathrm{Al}_{2} \mathrm{O}_{3}$-water nanofluid heated partially from side walls. - Alexandria Eng. J., vol.57, pp.1401-1412.

[8] Eiamsa-ard S. and Wongcharee K. (2018): Convective heat transfer enhancement using Ag-water nanofluid in a micro-fin tube combined with non-uniform twisted tape. - Int. J. Mech. Sci., vol.146-147, pp.337-354.

[9] Timofeeva E.V., Routbort J.L. and Singh D. (2009): A benchmark study on the thermal conductivity of nanofluids. J. Appl. Phy., vol.106, 094312.

[10] Murshed S.M.S., Nieto de Castro C.A., Lourenco M.J.V., Lopes M.L.M. and Santos F.J.V. (2011): A review of boiling and convective heat transfer with nanofluids. Renewable and Sustainable Energy Reviews, vol.15, No.5, pp.2342-2354.

[11] Das K., Duari P.R. and Kundu P.K. (2016): Effects of magnetic field on an unsteady mixed convection flow of nanofluids containing spherical and cylindrical nanoparticles. - J. Heat Trans., ASME, vol.138, No.6, pp.061901-7.

[12]. Mandy A. (2012): Unsteady mixed convection boundary layer flow and heat transfer of nanofluids due to stretching sheet. - Nuclear Engg. Design, vol.249, pp.248-255.

[13] Kundu P.K. and Sarkar A. (2017): Multifarious slips perception on unsteady Casson nanofluid flow impinging on a stretching cylinder in the presence of solar radiation. - Eur. Phys. J. Plus, vol.132, pp.144.

[14] Kai-Long Hsiao (2017): Micropolar nanofluid flow with MHD and viscous dissipation effects towards a stretching sheet with multimedia feature. - Int. J. Heat Mass Trans., vol.112, pp.983-990.

[15] Reddy P.S. and Chamkha A. (2017): Heat and mass transfer characteristics of MHD three-dimensional flow over a stretching sheet filled with water-based alumina nanofluid. - Int. J. Num. Methods Heat Fluid Flow, vol.28, No.3, pp.532-546.

[16] Das K., Sarkar A. and Kundu P.K. (2017): Cu-water nanofluid flow induced by a vertical stretching sheet in presence of a magnetic field with convective heat transfer. - vol.6, No.3, pp.206-2013.

[17] Gireesha B.J., Mahantheshc B., Thammanna G.T. and Sampathkumar P.B. (2018): Hall effects on dusty nanofluid two-phase transient flow past a stretching sheet using KVL model. - J. Mol. Liq., vol.256, pp.139-147. 
[18] Ghadikolaei S.S., Hosseinzadeh Kh., Yassari M., Sadeghi H. and Ganji D.D. (2018): Analytical and numerical solution of non-Newtonian second-grade fluid flow on a stretching sheet. - Therm. Sci. Eng. Prog., vol.5, pp.309316.

[19] Nayak M.K., Akbar N.S., Pandey V.S., Khan Z.H. and Tripathi D. (2017): 3D free convective MHD flow of nanofluid over permeable linear stretching sheet with thermal radiation. - Powder Tech., vol.315, pp.205-215.

[20] Valipour P., Moradi R. and Aski F.S. (2017): CNT-water nanofluid thermal radiation heat transfer over a stretching sheet considering heat generation. - J. Mol. Liq., vol.237, pp.242-246.

[21] Zeeshan A., Shehzad N., Ellahi R. and Sultan Z. Alamri (2018): Convective Poiseuille flow of $\mathrm{Al}_{2} \mathrm{O}_{3}$-EG nanofluid in a porous wavy channel with thermal radiation. - Neural Comput. Applic., vol.30, No.11, pp.33713382.

[22] Kumar K.G., Ramesh G.K., Gireesha B.J. and Gorla R.S.R. (2018): Characteristics of Joule heating and viscous dissipation on three-dimensional flow of Oldroyd B nanofluid with thermal radiation. - Alexandria Eng. J., vol.57, pp.2139-2149.

[23] Sheikholeslami M. and Rokni H.B. (2018): Numerical simulation for impact of Coulomb force on nanofluid heat transfer in a porous enclosure in presence of thermal radiation. - Int. J. Heat Mass Trans., vol.118, pp.823-831.

[24] Nayak M.K., Shaw S., Pandey V.S. and Chamkha A.J. (2018): Combined effects of slip and convective boundary condition on MHD 3D stretched flow of nanofluid through porous media inspired by non-linear thermal radiation. - Indian J. Phy., vol.92, No.8, pp.1017-1028.

[25] Daniel Y.S., Aziz Z.A., Ismail Z. and Salah F. (2018): Impact of thermal radiation on electrical MHD flow of nanofluid over nonlinear stretching sheet with variable thickness. - Alexandria Eng. J, vol.57, No.3, pp.21872197

[26] Qayyum S., Hayat T. and Alsaedi (2018): Thermal radiation and heat generation/absorption aspects in third grade magneto-nanofluid over a slendering stretching sheet with Newtonian conditions. - Physica B: Condensed Matter, vol.537, pp.139-149.

[27] Tiwari R.K. and Das M.K. (2007): Heat transfer augmentation in a two-sided lid-driven differentially heated square cavity utilizing nanofluids. - Int. J. Heat Mass Trans, vol.50, pp.2002-2018.

[28] Oztop H.F. and Abu-Nada E. (2008): Numerical study of natural convection in partially heated rectangular enclosers with nanofluids. - Int. J. Heat Fluid Flow, vol.29, pp.1326-1336.

[29] Xie H., Wang J., Xi T. and Liu Y. (2002): Thermal conductivity of suspensions containing nanosized SiC particles. - International Journal Thermophysics, vol.23, No.2, pp.571-580.

[30]. Murshed S.M.S., Leong K.C. and Yang C. (2005): Enhanced thermal conductivity of TiO2-water based nanofluids. - Int. Jour Therm. Sciences, vol.44, No.4, pp.367-373.

[31] Hamilton R.L. and Crosser O.K. (1962): Thermal conductivity of heterogeneous two component systems. Industrial and Engineering Chemistry Fundamentals, vol.1, No.3, pp.182-191.

[32] Cortell R. (2008): Radiation effects for the Blasius and Sakiadis flows with a convective surface boundary condition. - Appl. Math. Comput., vol.206, pp.832-840.

Received: March 25, 2019

Revised: November 12, 2019 